\title{
Speedup Techniques for Molecular Dynamics Simulations of the Interaction of Acoustic Waves and Nanomaterials
}

\author{
$\underline{\text { H.A. Bennett }}^{\text {a }}$, A.C. Zander ${ }^{\text {a }}$, B.S. Cazzolato ${ }^{\text {a }}$ and D.M. Huang ${ }^{\text {b }}$ \\ ${ }^{a}$ School of Mechanical Engineering, University of Adelaide, South Australia \\ ${ }^{b}$ Department of Chemistry, University of Adelaide, South Australia \\ Email: hywel.bennett@adelaide.edu.au
}

\begin{abstract}
Nanomaterials are seen to have great potential for use in the area of sound absorption. However, direct inspection of the interactions between acoustic waves and nanomaterials is not feasible due to the short time and length-scales involved. Molecular dynamics simulations can assist in improving understanding of the mechanisms involved in this process, but they have limitations that must be overcome to make their use viable.

The primary limitation is that molecular dynamics is computationally expensive, making the time-scales over which results can be obtained very short. This, in turn, makes the acoustic frequencies that can be examined extremely high. In the current work, the use of a simplified force field, multiple time-stepping, and an analytical description of the sound source producing the acoustic waves are investigated as methods to improve the speed of a model that simulates acoustic wave and nanomaterial interactions, as speedup directly translates into increased feasibility of longer time-scales (lower acoustic frequencies) and larger domains. The speedup and accuracy of these techniques are determined through benchmarking against existing computational results for the interaction of a carbon nanotube with a $2.57 \mathrm{GHz}$ acoustic wave propagating through argon gas.

Significant speedup is obtained using these techniques: replacing the oscillating atomistic wall in the benchmark case with the analytical oscillating wall produces a speedup factor of 1.3 ; using the simpler Dreiding force field for the carbon nanotube instead of the benchmark case's REBO potential results in a speedup factor of 3.6; and exchanging the Velocity Verlet time integrator in the benchmark case with an rRESPA multiple time-step integrator along with using the Dreiding force field leads to a speedup factor of approximately 39. Combining all of these techniques further increases the speedup, resulting in a speedup factor of approximately 50 compared with the benchmark. The error introduced into the numerical results is no greater than $6 \%$, suggesting these speedup techniques are appropriate for molecular dynamics simulations of acoustic wave and nanomaterial interactions.
\end{abstract}

Keywords: Molecular dynamics, nanomaterials, acoustics 


\section{INTRODUCTION}

Classical acoustics suggests that the acoustic absorption of a fibrous material improves as the fibres become finer (Bies and Hansen, 2009 p. 55). This implies that nanomaterials, which have extremely fine characteristic dimensions, may have the potential to be extremely good acoustic absorbers. Currently, the understanding of the acoustic absorption mechanisms present at the nanoscale is limited, with direct observation of phenomena not being feasible due to the nanomaterials being of such small size and the interactions varying rapidly with time. Simulations are better suited than experiments to investigate acousticwave-nanomaterial interactions but also possess limitations. Molecular dynamics (MD) has been identified as the most suitable method for simulating nanomaterial and acoustic wave interactions (Ayub et al., 2013), but is plagued by the problem of having a high computational cost. This high computational cost leads to limitations in the system size and duration that can be feasibly modelled, restricting the capabilities of such models. This work aims to improve the modelling capabilities through increasing the speed of these types of simulations by surveying existing techniques and verifying their applicability to acoustic wave interactions with nanomaterials. Increasing the speed at which the simulation runs should increase the domain size and length of time that can be modelled. The techniques that are investigated in this work are the substitution of an atomistically described sound source (Ayub et al., 2013) with an analytical potential function, use of a simpler force field, and multiple time-stepping. Once the feasibility of these techniques is established, this work also investigates the use of these techniques in conjunction. It is anticipated that the work presented here will be foundational in developing models capable of simulating nanomaterial and acoustic wave interactions for audible frequencies, which is currently prevented by time and length-scale limitations.

A benchmarking process is used to assess the approximate speedup that can be expected when each technique is applied to a simulation. The benchmark allows both the speedup and accuracy of the different techniques to be assessed. The speedup results are also indicative of which techniques provide the greatest improvement, and hence should be considered for use when modelling other acoustic wave and nanomaterial scenarios.

\section{BENCHMARK CASE}

The benchmark case selected as a baseline for comparison, to reflect current approaches, is the model devised by Ayub et al. (2014) and was selected as it is a molecular dynamics simulation specifically designed for inspecting carbon nanotube (CNT) and acoustic wave interactions. The benchmark uses the software LAMMPS (Plimpton, 1995) as the simulation package. The simulation box of the benchmark case (see Figure 1 ) is $70 \times 70 \times 150$ $\mathrm{nm}$, containing a 25-nm-long single-walled open-ended CNT attached perpendicular to a reflective wall at one end of the

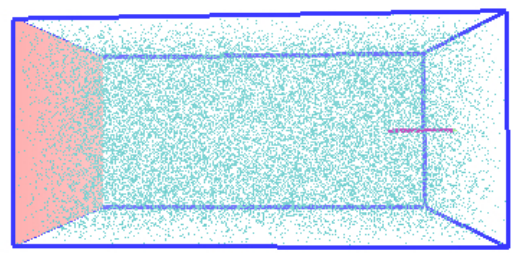

Figure 1. Benchmark simulation snapshot with CNT in purple (right) and atomistic piston in pink (left).

domain. The CNT is composed of 2,040 atoms and is surrounded by an argon gas medium. The gas, consisting of 20,407 atoms, propagates the acoustic wave generated by the sound source at the opposite end of the system to the CNT. The sound source consists of a single layer of a face centered cubic lattice of atoms with the same diameter as the argon atoms, oscillated sinusoidally with velocity amplitude of 0.50 angstrom per picosecond and $2.57 \mathrm{GHz}$ frequency to generate an acoustic wave. In the remaining text this sound source will be referred to as the 'atomistic piston', and is composed of 35,645 atoms. The atoms of the piston interact via a purely repulsive Weeks-Chandler-Andersen (WCA) potential (Weeks et al., 1971), which is equivalent to a Lennard-Jones 12-6 potential, with a cutoff radius of $2^{1 / 6}$ times the atom diameter. The bonded interactions between the carbon atoms of the CNT are described using the REBO (Reactive Empirical Bond Order) potential (Stuart et al., 2000), which has been widely used to model the mechanical properties of CNT, including by Białoskórski and Rybicki (2012). With REBO, the bonds between individual carbon atoms are not pre-defined; instead they are constructed based on the local environment of the atoms. All the pair interactions are modelled using a Lennard-Jones 12-6 potential (Allen and Tildesley, 1989 p. 9),

$$
\Phi(r)=4 \varepsilon\left\lfloor\left(\frac{\sigma}{r}\right)^{12}-\left(\frac{\sigma}{r}\right)^{6}\right\rfloor
$$

where $r$ is the distance between the two atoms, and the interaction parameters detailed in Table 1 are the energy parameter, $\varepsilon$, and the diameter, $\sigma$. This potential is applied when $r$ is less than the cutoff radius, $r_{c}$, and shifted such that the potential is zero at $r=r_{c}$. To allow the energy supplied by the piston to be dissipated, the system's temperature is maintained at $273 \mathrm{~K}$ using a Nosé-Hoover NVT thermostat (Shinoda et al., 2004). Periodic boundary conditions are applied to the long sides of the simulation box, such that the model acts as if the span of the piston were infinite in the $x$ and $y$ directions, and there are infinitely many CNTs, spaced at 
the domain width, forming an array. The wall to which the CNT is tethered is defined as being specular, representing the presence of the rigid substrate. The benchmark case uses a 0.5 fs time-step and Velocity Verlet time integrator, running on two computing cluster nodes each comprising four AMD 6238 12-core 2.6 $\mathrm{GHz}$ CPUs for a total of 96 processors, with a high-speed QDR InfiniBand network connecting the nodes. Unless stated otherwise, the simulations use the same specifications as the benchmark, for consistency.

Table 1. Lennard-Jones parameters used in benchmark case (Based on Carlborg et al. (2008)).

\begin{tabular}{|ccccc|}
\hline \multicolumn{2}{|c}{ Interaction Pairs } & Energy Parameter, $\varepsilon\left(10^{-3} \mathrm{eV}\right)$ & Diameter, $\sigma(\AA)$ & Cutoff Radius, $r_{\mathrm{c}}(\AA)$ \\
\hline $\mathrm{Ar}$ & $\mathrm{Ar}$ & 10.33 & 3.4 & 10.0 \\
\hline $\mathrm{Ar}$ & $\mathrm{C}$ & 4.98 & 3.38 & 11.9 \\
\hline Piston & $\mathrm{Ar}$ & 10.33 & 3.4 & 3.816 \\
\hline Piston & $\mathrm{C}$ & 4.98 & 3.38 & 3.794 \\
\hline
\end{tabular}

\section{METHOD OF ANALYSIS}

To assess the accuracy of the acoustic simulations when using the different techniques the velocity profiles of the systems are compared, considering the velocities of the simulated gas atoms relative to their position along the length of the simulation box, after being decomposed into cosine and sine components. These components were calculated based on a standing wave of the form (Hadjiconstantinou \& Garcia, 2001)

$$
v(z, t)=A(z) \sin (\omega t)+B(z) \cos (\omega t)
$$

where $t$ and $\omega$ are the time and acoustic frequency, respectively, and $A(z)$ and $B(z)$ are coefficients of the sine and cosine components of the velocity, which depend on the distance along the simulation domain, $z$, and are extracted using a least-squares method. These profiles were used to compare the propagation of the acoustic wave through the gaseous medium, thereby allowing the gas-gas interactions and gas-piston interactions to be assessed, as major deviations in either would result in changes to the velocity profile. The temperature of the gas is also a useful tool when comparing the systems, allowing the average kinetic energy of the gas particles with respect to time to be analysed and the energy imparted to the gas, which is dependent on the motion of the piston, to be compared with the benchmark. The kinetic energy of the CNT is also a useful metric for comparison, allowing the investigation of whether the forces imposed on the CNT and bonded interactions within the CNT, and hence net motion, are comparable to those found in other models.

The speedup of the various techniques was quantified by calculating a 'speedup factor', the ratio of the CPU time of the benchmark case to the CPU time of the new system. Using CPU time is more appropriate than wall-time as it indicates the gains that could be made when running several simulations concurrently, with limited available hardware. This is important for the type of applications where acoustic-wave-nanomaterial interaction simulations would be used, including calculating acoustic absorption curves for a nanomaterial. The absorption curve would require a number of frequencies to be investigated and hence a number of separate simulations to be run for each for each frequency, thus optimising use of available hardware for running multiple simulations is important. Though the CPU time has been derived from a single run of each model, each run consisted of millions of time-steps, and covers multiple acoustic wave periods, which is sufficient to limit stochastic variations in run-time intrinsic to the simulation.

\section{ANALYTICAL PISTON}

In the benchmark simulation the sound source is modelled using an oscillating atomistic piston, which can be thought to represent a driven rigid loudspeaker cone. This piston, however, is comprised of an extremely large number of atoms, over half the atoms of the system, indicating that an alternate formulation for the sound source may be more efficient and considerably reduce the run-time of the simulation. Replacing the atomistic piston with an analytical wall is of particular interest, as it would remove the need to model the piston atoms, while maintaining a potential and motion equivalent to the atomistic piston. The most appropriate potential was considered to be the Lennard-Jones 9-3 (LJ9-3) potential, which can be derived by integrating a half-lattice of Lennard-Jones 12-6 (LJ12-6) atoms in three dimensions, due to the piston atoms in the benchmark possessing an LJ12-6 potential. It is important to note that, though a good choice, the LJ9-3 wall does not represent the benchmark case with complete accuracy; firstly, the LJ9-3 potential is derived using an LJ12-6 potential that has no cut-off radius. Secondly, the atomistic piston is not a half-lattice, with the lattice extending infinitely beyond the wall position, but consists of a single layer of a lattice, such that by definition it is not portrayed accurately by the LJ9-3 potential. Realistically, however, sound sources such as a speaker would not have a single layer thickness, and that a half-lattice is more representative of an 
experimental scenario. To ensure the region of influence of the analytical piston did not extend beyond that of the atomistic piston, the same cutoff distance was chosen for the analytical potential. This spatially averaged potential also has the benefit of reducing the number of interactions that need to be calculated, as contributions from all the piston atoms have already been summed together in the LJ9-3 potential.

The speedup obtained, however, was not equivalent to the percentage decrease in the system's number of atoms. Due to the number of interactions with the piston being limited compared with those of the entire system, the speedup from substituting the atomistic piston with the analytical piston was only approximately 1.3 , although the piston contained half the atoms of the system, it did not participate in half the interactions.

The temperature of the gas (see Figure 2) suggests the gas interaction with the two piston types is similar, with the mean temperature only differing by approximately $1 \mathrm{~K}$, and the temperature variations being of a similar size and period. These variations are associated with the oscillation of the pistons, which were the same in both models. The similarities are somewhat masked by a low frequency modulation of the mean which appears to be from the stochastic nature of the problem. The velocity profile of the analytical piston simulation is similar in shape to the benchmark, though does feature some differences in magnitude (see Figure 3). These errors would appear to be due to the analytical piston being representative of a

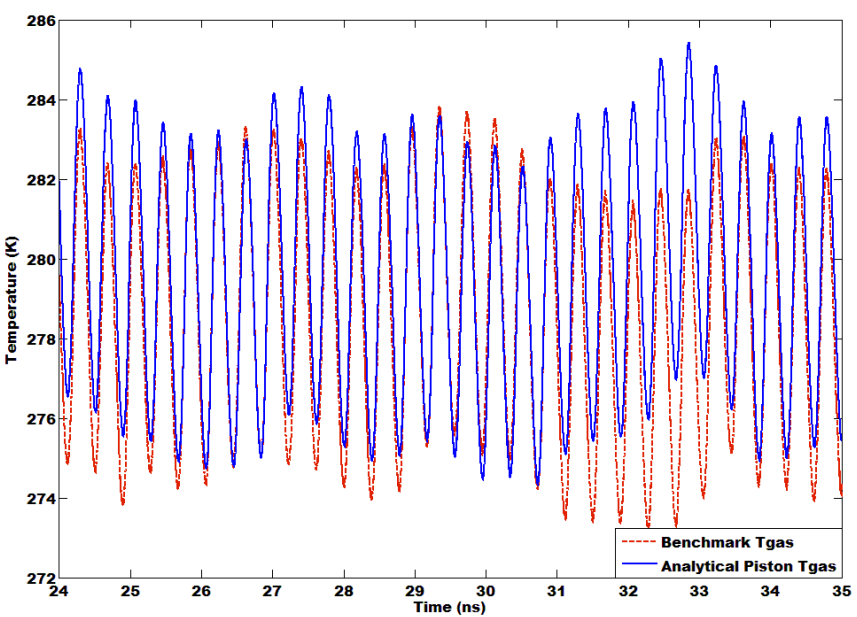

Figure 2. Gas temperature comparison for benchmark and analytical piston cases.

half-lattice, rather the planar lattice of the benchmark case, and are considered acceptable. This indicates that the analytical wall is a suitable alternative to the atomistic piston.

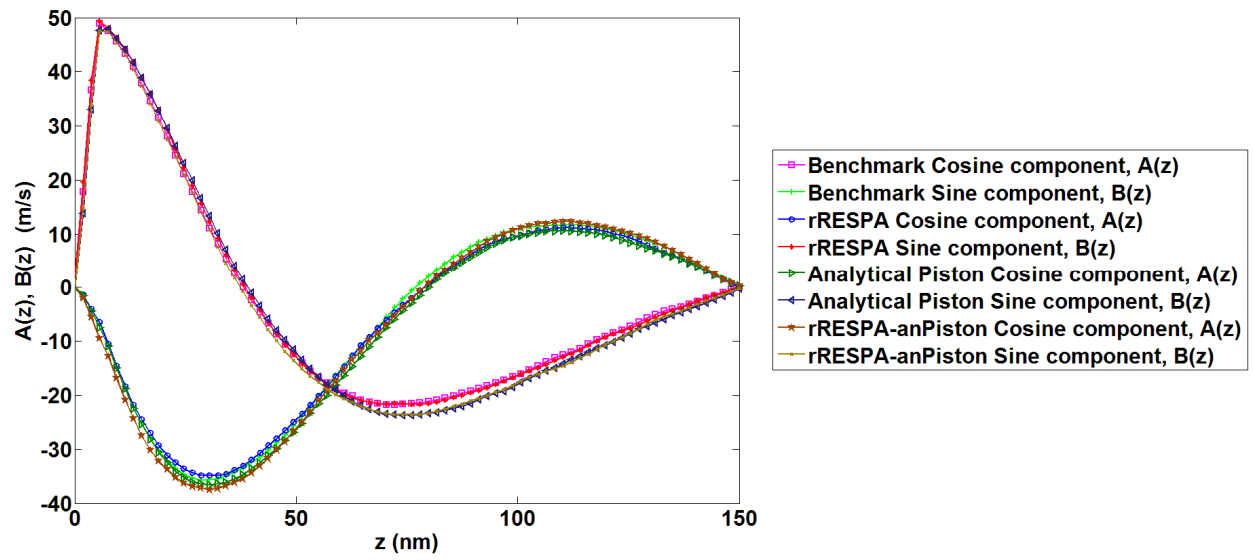

Figure 3. Velocity profile comparison for analytical piston, rRESPA and combined model.

\section{SIMPLER CNT FORCE FIELD}

Using a simpler force field to describe the interactions in the system also has potential to generate speedup, as calculating simpler interactions will take less time. However, the alternate force field needs to be appropriate if correct results are to be produced. Hence, the Dreiding force field, developed by Mayo et al. (1990), was selected for investigation as an alternative set of potentials, since it has previously been used for describing carbon nanotubes and predicting their vibrational behaviour (Chen et al., 2011). As only carboncarbon bonds occur in the simulation, only one set of parameters is required to describe each of the finer level interactions of the simulation. Here, the parameters used by Chen et al. (2011) were used to describe the bond, angle and dihedral interactions. The bond interactions use a Morse potential

$$
E_{B}=D\left[1-e^{-\alpha\left(r-r_{0}\right)}\right]^{2}
$$


with an energy parameter $D$ of $4.964 \mathrm{eV}$, stiffness parameter $\alpha$ of $2.1867 \AA^{-1}$ and equilibrium bond distance $r_{0}$ of $1.418 \AA$. The bond angle interactions use a cosine-squared potential

$$
E_{A}=K_{A}\left[\cos (\theta)-\cos \left(\theta_{0}\right)\right]^{2}
$$

with a prefactor $K_{A}$ of $2.9084 \mathrm{eV}$ and equilibrium angle $\theta_{0}$ of $120^{\circ}$. The dihedral interactions use a CHARMM force field description

$$
E_{D}=K_{D}[1+\cos (n \phi-d)]
$$

with energy coefficient $K_{D}$ of $0.1302 \mathrm{eV}$, multiplicity $n$ of 2 and phase $d$ of $180^{\circ}$. As gas-gas and gas-CNT interactions are likely to be dominated by repulsive interactions, the Dreiding force field was modified by replacing the Lennard-Jones pair potentials with WCA potentials with the same diameters and energy parameters. The results of Figure 4 indicate using such a force field agrees with the benchmark results.

The analysis of the kinetic energy of the carbon nanotube (in Table 2) shows that there is agreement between the benchmark and Dreiding models, with the mean and standard deviation being within two and five per cent, respectively, and the standard error being small, giving confidence in the mean value calculated. This indicates a level of consistency between the way the carbon nanotube is described by the REBO potential and Dreiding force field, and that the Dreiding force field is appropriate for use in further simulations. Use of the modified Dreiding force field, where the pair interactions use a WCA potential, is further supported by the velocity profile being similar to the benchmark, suggesting that the gas interactions, i.e., the pair interactions with argon, are reasonably consistent with those present in the benchmark case. Hence the use of the WCA potential is considered suitable for this type of simulation as it does not significantly alter the characteristics of the gas. Therefore, the modified Dreiding force field is a feasible option for modelling acoustic-wavenanomaterial simulations. Due to the relative simplicity of the Dreiding force field compared with the REBO potential, the model using the modified Dreiding force field produces a speedup factor of 3.6 compared with the benchmark, a considerable speedup for one technique.

It was also thought prudent to check the maximum time-step that could be used while retaining accuracy. It was determined that carbon double-bond stretching has a period of $20 \mathrm{fs}$, based on data from Ouellette and Rawn (2015, p. 427). To allow the interactions to be correctly described, a time-step an order of magnitude smaller, at $2 \mathrm{fs}$, was selected for investigation, as a theoretical upper bound for the time-step. The results of the 2 fs time-step simulation using the Dreiding force field are included in the velocity profile of Figure 4 and the CNT kinetic energy comparison in Table 2.

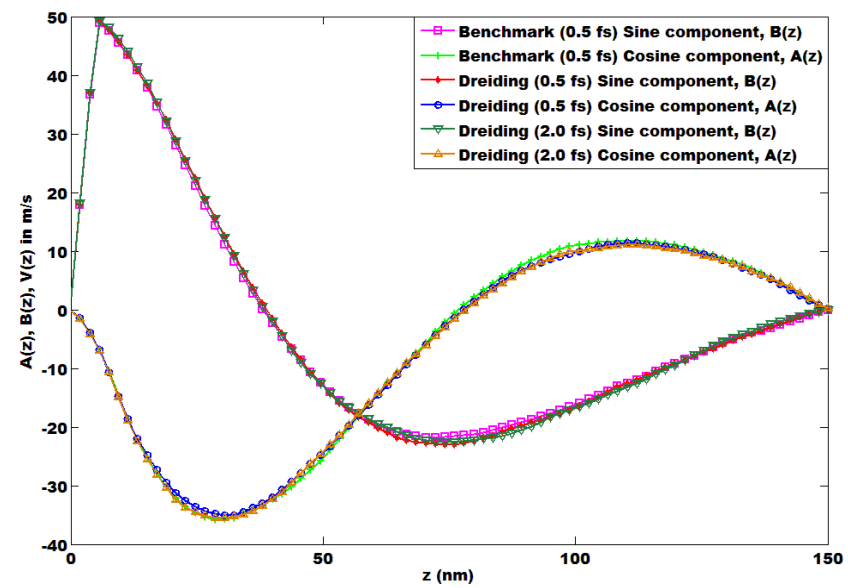

Figure 4. Velocity profile comparison of benchmark and Dreiding cases.
It is clear from Table 2 that the accuracy of the results decrease slightly as the time-step increases, with the 2 fs Dreiding case having errors of around five and eight percent, for the mean and standard deviation of the kinetic energy, respectively. The difference in the mean suggests that it would be prudent to use a time-step shorter than 2 fs in future simulations. Though a decrease in accuracy occurs, a speedup close to the factor by which the time-step was increased is provided by the change in time-step. Hence, a speedup factor of 13.9 was achieved by the 2 fs Dreiding case, compared with the benchmark case.

\section{MULTIPLE TIME-STEPPING}

Multiple time-stepping has been known to decrease simulation run-time in a number of situations, by virtue of reducing the number of calculations performed. The mechanism by which this is performed involves dividing simulation propagation into a number of levels, in this case by type of interaction, with each level being time integrated with a different frequency. This allows interactions that vary more slowly in time to be calculated less frequently, avoiding redundant calculations. Consequently, bond interactions at the finest level have a shorter time-step than the pair interactions at the coarsest level, which vary more slowly with time. The proportions of different atom types in the system under consideration here and, hence, the 
distribution of interactions in the simulation strongly indicate the benefits of using multiple time-stepping, as the majority of the atoms are monatomic argon that only undergo slowly varying pair interactions, while a relatively small proportion of atoms are carbon and are subjected to bond, bond angle, and bond dihedral interactions, which are calculated more frequently. The ratio of the time-step of one level to an adjacent level is called the loop factor, e.g. the loop factor between 2 fs and 1 fs time-step levels would be two. The multiple time-stepping technique used in this work is the rRESPA (reversible reference system propagation algorithm) integrator developed by Tuckerman et al. (1992).

Due to incompatibility between the REBO potential and rRESPA, the Dreiding force field was used for the multiple time-stepping simulations. Given the accuracy of the results from the 2 fs Dreiding simulations, a time-step of $1.25 \mathrm{fs}$ for the finest level was selected for the rRESPA simulations. A loop factor of two was chosen for all adjacent levels, leading to time-steps of 2.5, 5.0, and 10.0 fs for the bond angle, dihedral and pair levels, respectively. Subject to a modified Dreiding force field and these parameters, the model produced the results given in Figure 3 and Table 2 for the velocity profile and CNT kinetic energy, respectively, when using the rRESPA integrator. It is clear from the benchmark and rRESPA results in Figure 3 being almost coincident that the multiple time-stepping technique has not introduced significant error to the gas interactions. Furthermore, the standard error is still small and the discrepancy compared to the benchmark for both the mean and standard deviation of the kinetic energy of the CNT are less than five percent for this set of parameters, indicating their suitability, while a speedup factor of approximately 21 was achieved.

Further improvement was also attained through changing the number of processors. As communication was found to be excessive in contribution to the rRESPA simulation's run-time, at $70 \%$ of the total time, the number of processors was halved, such that one node was used. The resultant wall-time was found to be similar to the previous case, but the CPU-time almost halved, leading to a speedup factor of approximately 39. Further halving the number of processors was found to be less

Table 2. Comparison of kinetic energy of CNT for Dreiding and rRESPA models in eV.

desirable as the increase in wall-time was more pronounced, since the inter-node communication overhead was already removed in the reduction to one node. Such improvement may not be present for different computer architectures, but the findings indicate that selection of the number of nodes should not be arbitrary when efficiency is a major concern, and may have a considerable impact on the CPU time of the simulation.

\section{COMBINED rRESPA-ANALYTICAL PISTON MODEL}

As the speedup from different techniques can be cumulative, the use of the documented techniques in conjunction with one another was investigated. Combining the analytical piston with the rRESPA model using one node of processors, a speedup factor of 50 was achieved. Both the velocity profile and kinetic energy of the CNT agree with the benchmark results, with the velocity profile closely resembling the analytical piston results (see Figure 3). Compared with the benchmark results, a maximum error of approximately $5 \%$ is present in the velocity profile, much of which is inherited from the analytical piston technique, while the mean kinetic energy was found to have an error of approximately $5.8 \%$. These results are considered acceptable due to the significant speedup provided by the combined model, such that it can be suggested that the model is suitable for the modelling of nanomaterial and acoustic wave interactions.

\section{DISCUSSION AND CONCLUSION}

It is clear from the speedup that has been achieved in the aforementioned simulations, and collated in Table 3 , that there is great potential for speedup techniques, such as rRESPA and an analytical formulation of the sound source, to be used in MD models of nanomaterial-acoustic-wave interactions. A speedup factor of 50 is a significant improvement, and will permit a noticeable increase in both the time and length-scales of the simulations that may be performed, allowing larger nanomaterial structures and lower acoustic frequencies to be studied through MD modelling. If not significantly changing the CNT size, modelling a frequency an order of magnitude lower than current should be feasible. Lower frequency models will mainly involve running longer simulations, which does not alter the molecular-level physical processes being modelled. Hence the same accuracy captured at high frequencies is expected to occur in lower frequency simulations using the same time-step and interaction parameters. The techniques examined here are a good starting point for further improvements, as they are grounded in the selection of potentials and time integrator, and hence, should be easily applied to nanomaterial-acoustic-wave simulations beyond the benchmark scenario. The 
ability of different speedup techniques to complement one another was also demonstrated, such that small gains by individual techniques may be combined to obtain more sizable speedup factors. Although significant speedup has been realised, allowing behaviour at lower frequencies to be examined, accurate simulation of nanomaterial-acoustic-wave interactions at audible frequencies remains a challenge. To permit modelling of audible frequencies it is expected that speedup techniques in conjunction with hybrid, continuum and noncontinuum models will be required, and is the subject of current research.

Table 3. Summary of techniques and speedup obtained.

\begin{tabular}{|cccc|}
\hline Technique & Time-Step (fs) & Number of Processors & Benchmarked Speedup Factor \\
\hline Analytical Piston & 0.5 & 96 & 1.3 \\
\hline Modified Dreiding force field & 0.5 & 96 & 3.6 \\
\hline Modified Dreiding force field & 2.0 & 96 & 13.9 \\
\hline rRESPA & 10.0 & 96 & 21.0 \\
\hline rRESPA & 10.0 & 48 & 39.2 \\
\hline rRESPA-Analytical Piston & 10.0 & 48 & 50.8 \\
\hline
\end{tabular}

\section{ACKNOWLEDGMENTS}

This research was supported by the Australian Research Council's Discovery Projects funding scheme (project number DP 130102832). The authors would like to thank Mr. Md Ayub for assistance with the benchmark simulation and eResearch SA for computational resources.

\section{REFERENCES}

Allen, M.P. and Tildesley, D.J. (1989). Computer Simulation of Liquids. Oxford University Press.

Ayub, M., Zander, A.C., Howard, C.Q., Cazzolato, B.S. and Huang, D.M. (2013). A review of MD simulations of acoustic absorption mechanisms at the nanoscale, Acoustics 2013, Australian Acoustical Society, Gold Coast, Australia, 2-4 November.

Ayub, M., Zander, A.C., Howard, C.Q., Huang, D.M. and Cazzolato, B.S. (2014). Molecular dynamics simulations of sound wave propagation in a gas and thermo-acoustic effects on a carbon nanotube, International Conference on Theoretical and Computational Acoustics, Texas, USA, 10-14 March.

Białoskórski, M. and Rybicki, J. (2012). Mechanical properties of single-walled carbon nanotubes simulated with AIREBO force-field. Computational Methods in Science and Technology, 18(2), 67-77.

Bies, D.A. and Hansen, C.H. (2009). Engineering Noise Control: Theory and Practice. 4th edn, Spon Press, Abindon.

Carlborg, C.F., Shiomi, J. and Maruyama, S. (2008). Thermal boundary resistance between single-walled carbon nanotubes and surrounding matrices. Physical Review B. 78(20), 205406.

Chen, C., Ma, M., Jin, K., Liu, J. Z., Shen, L., Zheng, Q. and Xu, Z. (2011). Nanoscale fluid-structure interaction: Flow resistance and energy transfer between water and carbon nanotubes. Phys. Rev. E, 84(4), 046314.

Hadjiconstantinou, N.G. and Garcia, A.L. (2001). Molecular simulations of sound wave propagation in simple gases. Physics of Fluids, 13(4), 1040-1046.

Mayo, S.L., Olafson, B.D. and Goddard, W.A. (1990). DREIDING: a generic force field for molecular simulations. Journal of Physical Chemistry. 94(26), 8897-8909.

Ouellette, R.J. and Rawn, J.D. (2015). Principles of Organic Chemistry. Elsevier, USA.

Plimpton, S.J. (1995). Fast parallel algorithms for short-range molecular dynamics, Journal of Computational Physics, 117, 1-19.

Shinoda, W., Shiga, M. and Mikami, M. (2004). Rapid estimation of elastic constants by molecular dynamics simulation under constant stress. Phys. Rev. B, 69(13), 134103.

Stuart, S.J., Tutein, A.B. and Harrison, J.A. (2000). A reactive potential for hydrocarbons with intermolecular interactions. The Journal of Chemical Physics. 112(14), 6472-6486.

Tuckerman, M., Berne, B.J. and Martyna, G.J. (1992). Reversible multiple time scale molecular dynamics. The Journal of chemical physics, 97(3), 1990-2001.

Weeks, J.D., Chandler, D. and Andersen, H.C. (1971). Role of repulsive forces in determining the equilibrium structure of simple liquids, The Journal of Chemical Physics, 54(12), 5237-5247. 